\title{
Metodología de doble evaluación modificada mediante la integración de entornos virtuales para el proceso de enseñanza y aprendizaje: Aplicación a la asignatura Teoría de Control del Grado en Ingeniería en Energía en la Universidad de Guadalajara (México)
}

\section{Jesús Águila-León ${ }^{\mathrm{a}}$, Carlos Vargas-Salgado ${ }^{\mathrm{b}}$, Cristian Chiñas-Palacios ${ }^{\mathrm{c}}$ y Paula Bastida-Molina $^{\mathrm{d}}$}

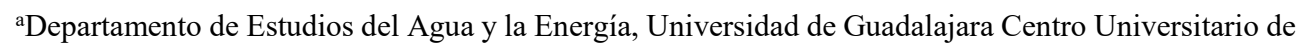
Tonalá, Jalisco, México, jesus.aguila@academicos.udg.mx, bepartamento de Ingeniería Eléctrica. Camino de Vera, s/n Edificio 5E, Universitat Politècnica de València, España, carvarsa@upvnet.upv.es, 'Departamento de Estudios del Agua y la Energía, Universidad de Guadalajara Centro Universitario de Tonalá, Jalisco, México, daniel.chinas@academicos.udg.mx e dinstituto Universitario de Ingeniería Energética, Camino de Vera s/n, Edificio 8E, 2a planta, Universitat Politècnica de València, España, paubasmo@etsid.upv.es.

\begin{abstract}
This paper presents a modification to the methodology of double evaluation (DDE, from the Double Evaluation in Spanish language), where the student, in addition of reinforcing his learning and developed competences, becomes a participant in the evaluation process by adding a self-assessment process paper-based for the first evaluation, as by means of an online form based on Google Classroom for the second evaluation of the modified DDE methodology. A comparative analysis of the improvement of student notes between a control group and a group to which the modified DDE methodology was applied is carried out. The results show that the group of the modified DDE methodology had a better performance and managed to apply more effectively the competences evaluated for the second tests, in addition the student achieved a deeper state of self-criticism thanks to the self-assessment mechanisms added to the methodology.
\end{abstract}

Keywords: double evaluation, competences, control theory, methodology, learning, virtual environment, Google Classroom.

\footnotetext{
Resumen

Este trabajo presenta una modificación a la metodología de doble evaluación (DDE), la cual consiste en una primera evaluación mediante la aplicación de una prueba de control, luego se realiza una realimentación sobre los resultados obtenidos y posteriormente se aplica una segunda evaluación. En el presente trabajo se ha añadido un mecanismo de autoevaluación donde el alumno, además de reforzar el aprendizaje y las competencias desarrolladas, participa en un proceso de autocrítica, favoreciendo la comprensión y el pensamiento crítico. La autoevaluación consta tanto de un formato fisico para
} 
la primera evaluación, como por medio de una evaluación en línea basada en Google Classroom para el caso de la segunda evaluación de la metodología DDE modificada. Posteriormente, se lleva a cabo un análisis comparativo de la mejora de resultados de las notas entre un grupo de control y un grupo al que se aplica la metodología DDE modificada. Los resultados muestran que el grupo de la metodología DDE modificada tuvo un mejor desempeño y logró aplicar de manera más efectiva las competencias evaluadas para las segundas pruebas, debido a una mejora en el análisis y la resolución de problemas.

Palabras clave: doble evaluación, competencias, teoría de control, metodología, aprendizaje, entorno virtual, Google Classroom.

\section{Introducción}

La Universidad de Guadalajara Centro Universitario de Tonalá ofrece estudios para la obtención de grado en Ingeniería en Energía. Parte de la materia que el alumno debe cubrir incluye asignaturas de cálculo diferencial, integral, modelado matemático, teoría y sistemas de control. Se espera que el alumno de Ingeniería en Energía complemente su formación con las competencias propias de estas asignaturas.

La asignatura de Teoría de Control se centra en el desarrollo de competencias y habilidades básicas de modelado matemático y análisis de estabilidad de sistemas. Esta asignatura se cursa de manera regular durante el sexto semestre del grado en Ingeniería en Energía. Las competencias básicas para el modelado matemático pueden agruparse en cinco principales categorías (Maßß, 2006):

1. Competencias para entender el problema de la realidad, y para establecer las condiciones de un modelo matemático basado en la realidad.

2. Competencias para crear un modelo matemático desde el problema real.

3. Competencias para solucionar problemas o cuestiones empleando el modelo matemático obtenido.

4. Competencias para interpretar los resultados obtenidos del modelo matemático con una situación de la realidad.

5. Competencias para validar la solución obtenida.

La metodología de la doble evaluación DDE, ayuda a reforzar las competencias anteriores. Dicha metodología consiste en la aplicación de dos pruebas para la evaluación de cada examen parcial de una asignatura. La primera prueba funciona como un primer control, luego del cual el profesor establece estrategias para subsanar las carencias detectadas mediante una sesión de realimentación. Posteriormente, se aplica una segunda evaluación y se evalúa la evolución del desempeño de los estudiantes. A nivel de ingeniería existen carencias importantes en conocimientos y competencias relacionadas con las matemáticas por parte de los estudiantes. Es por ello que buscar metodologías y herramientas que ayuden al estudiante a subsanarlas es muy importante. 
El proceso de enseñanza y aprendizaje de asignaturas que involucran el modelado matemático, tales como lo es Teoría de Control, conllevan un especial reto docente, al considerar las deficiencias típicas relacionadas a las asignaturas relacionadas con las matemáticas, donde el profesor ha de seguir una metodología adecuada a los objetivos del curso (Ang, 2001; Kaiser, 2011). Una de las principales dificultades que el docente enfrenta, además de la falta de interés, disgusto e incluso indiferencia hacia las matemáticas por parte de los alumnos, es la carencia de competencias y habilidades adecuadamente desarrolladas para el nivel que requiere una carrera en ciencias e ingeniería (Rylands \& Coady, 2009).

Se han hecho múltiples esfuerzos por desarrollar y mejorar metodologías para subsanar estas carencias (Greefrath \& Vorhölter, 2016), desde la creación de un marco trabajo bien estructurado que permita al estudiante desarrollar todas las competencias y habilidades necesarias para el modelado matemático (Eliciting, 2018; Keng C, 2005; Zawojewski, 2007) hasta el establecimiento de metodologías efectivas para la evaluación de dichas competencias (Zawojewski, 2007) así como de mecanismos de evaluación y retroalimentación que permitan al estudiante mejorar su desempeño por cuenta propia. Los autores (Boigues Planes, Estruch, Vidal, \& Pastor, 2018) presentan en su trabajo una propuesta de doble evaluación para la asignatura de Matemáticas del Grado de Ciencias Ambientales de la Universitat Politècnica de València.

Un aspecto importante a considerar en el desempeño de los alumnos es el hecho de que pertenecen a una generación de jóvenes inmersos en las tecnologías de la información y la computación (TICs), donde la interconectividad se aplica en todos los campos. Las metodologías de enseñanza deberían de adecuarse a la realidad de nuestros jóvenes, y echar mano de las ventajas de herramientas y entornos virtuales para facilitar el proceso de enseñanza y aprendizaje (Gargallo, García, \& Morera, 2015; Yavich \& Starichenko, 2017) donde no solo se trata de una plataforma digital dónde entregar las tareas y actividades, sino que se diseña pensando en un enfoque pedagógico que involucre herramientas que permitan una adecuada realimentación sobre resultados logrados por los estudiantes (Superior \& Superior, 2016) de tal manera que se incentive su mejora.

Dentro de los entornos virtuales más populares para gestión de asignaturas se encuentran Google Classroom y Moodle, existiendo también plataformas propias desarrollados por las universidades como es el caso de PoliformaT en la Universitat Politècnica de València. Estos entornos virtuales permiten desde compartir información, asignar y evaluar tareas y exámenes hasta crear foros de discusión sobre los temas de clase así como dar cursos a distancia (UPV, 2018). Por lo general los profesores y estudiantes ven a estos gestores más como un complemento a su clase (Abid Azhar \& Iqbal, 2018; Al-Maroof \& Al-Emran, 2018) sin considerar su impacto en la metodología de su clase. Sin embargo, aplicadas adecuadamente pueden ser complementos metodológicos importantes (Heggart \& Yoo, 2018). En este trabajo se propone la implementación de una metodología modificada DDE y la integración de un entorno virtual de Google Classroom con el objetivo de analizar la mejora en el proceso de enseñanza y aprendizaje en alumnos de pregrado. 


\section{Objetivos}

Aplicar la metodología de la doble evaluación modificada y comprobar si dicha metodología integrada a un entorno virtual de administración de la clase y seguimiento del progreso del alumno produce una mejora del proceso de enseñanza y aprendizaje para los estudiantes de la asignatura de Teoría de Control del grado en Ingeniería en Energía de la Universidad de Guadalajara. Se analizan también los resultados obtenidos con la metodología modificada DDE contra la metodología DDE propuesta por (Boigues Planes et al., 2018).

\section{Desarrollo de la innovación}

\subsection{Descripción y criterios}

Se propone una metodología modificada basada en la DDE, de doble evaluación, propuesta por (Boigues Planes et al., 2018), modificando el proceso de evaluación, integrando además un entorno virtual, en este caso la suite de Google Classroom, por ser una de las plataformas más conocidas y de uso público, aunque se podría aplicar a cualquier otra plataforma. Se toma como caso de estudio la asignatura de Teoría de Control para evaluar la metodología DDE.

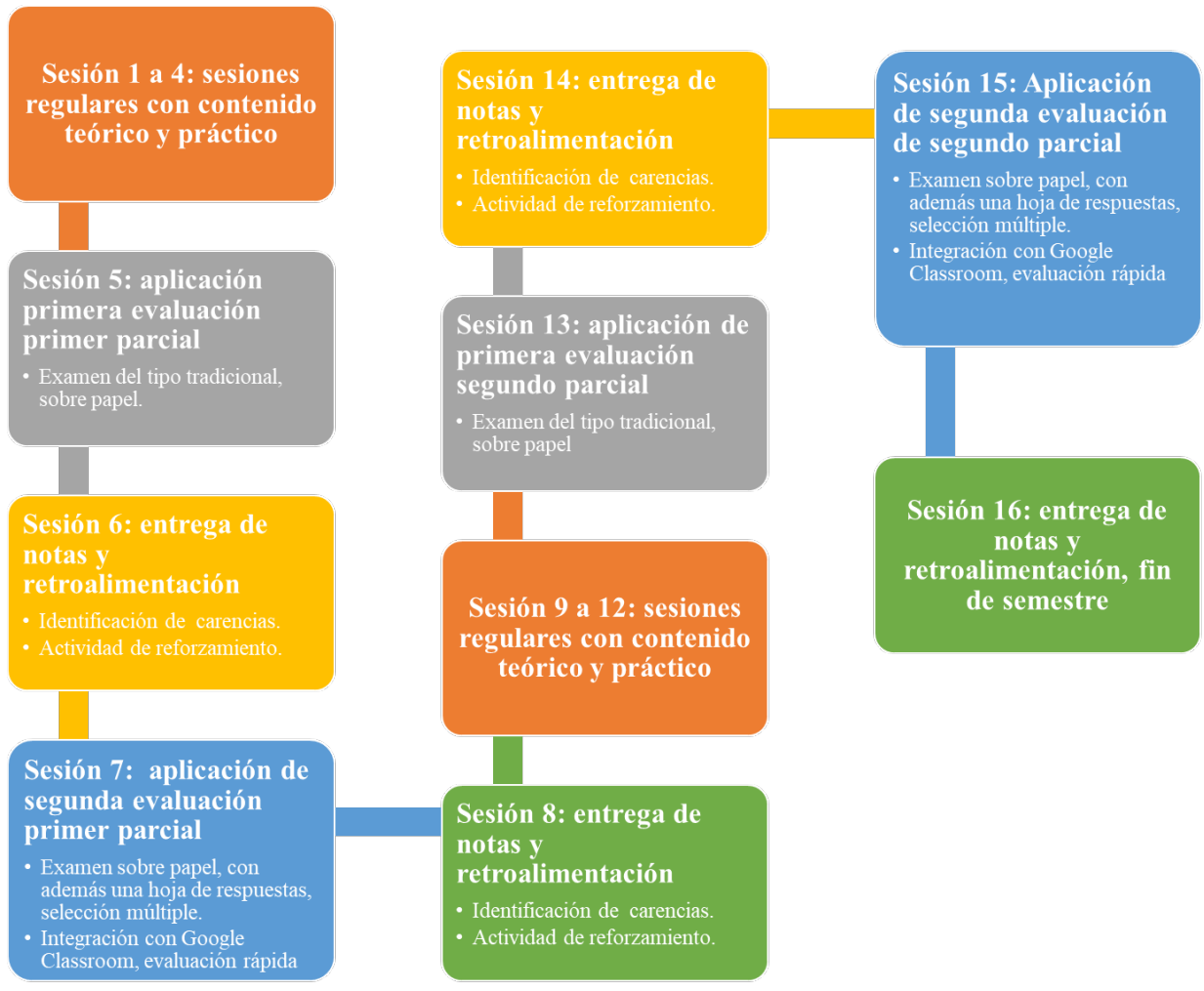

Fig. 1 Distribución propuesta de sesiones y evaluaciones a lo largo del curso

El programa de la asignatura se ha dividido en 16 sesiones, una por semana, que combinan contenidos teóricos y prácticos. El curso contempla la evaluación con dos exámenes 
parciales. Cada parcial se divide en dos actos de evaluación, con fechas distintas, donde se evalúan los mismos temas. El primer acto de evaluación se realiza del modo tradicional añadiendo el proceso de autoevaluación durante la sesión de realimentación posterior a la aplicación de dicho primer acto. En el segundo acto se aplica la metodología de la doble evaluación modificada en combinación de un formulario online. La Figura 1 muestra las principales etapas de la metodología planteada, así como su distribución temporal.

En el trabajo presentado por (Boigues Planes et al., 2018) la calificación final para cada parcial del cuatrimestre se obtiene considerando el mejor resultado de las dos evaluaciones según la Ecuación 1.

\section{Calificación parcial = max「evaluación1, evaluación 2$\rceil$}

Es decir, independientemente del resultado obtenido por el estudiante en cualesquiera de sus dos evaluaciones, se toma como nota final la nota más alta.

El utilizar la Ecuación 1 para evaluar los resultados de los estudiantes permite que el alumno tenga un incentivo para mejorar su nota, aun cuando haya obtenido una buena calificación en la primera evaluación, de cara a mejorar en la segunda evaluación. El principal aporte de la modificación de la metodología DDE presentado en este trabajo ha sido añadir los siguientes aspectos:

- Un acto de auto evaluación durante la sesión de retroalimentación, donde el estudiante se evalúa y se pone una nota en función de la evaluación.

- Un acto de auto calificación durante el segundo acto de evaluación, mediante la captura de respuestas, por parte de cada estudiante, en un formulario online de Google Classroom.

El integrar estos dos puntos anteriores a la metodología DDE hace que la metodología incentive al estudiante a mejorar para obtener una mejor nota en la segunda evaluación, subsanando sus carencias mediante el proceso de autocrítica y la retroalimentación dada por el profesor. Además, la metodología DDE modificada permite hacer el proceso de evaluación doble más dinámico y rápido para la obtención de las notas de los estudiantes, puesto que estos se vuelven partícipes del proceso mediante la autoevaluación en papel y el formulario online de Google Classroom.

\subsection{Integración de Google Classroom a las evaluaciones}

Como se mencionó con anterioridad, la plataforma de Google Classroom puede ser una herramienta poderosa para la metodología de enseñanza. Se proponen dos esquemas diferentes de evaluación para cada parte del curso. La Figura 2 muestra una fotografía de la primera evaluación aplicada. 

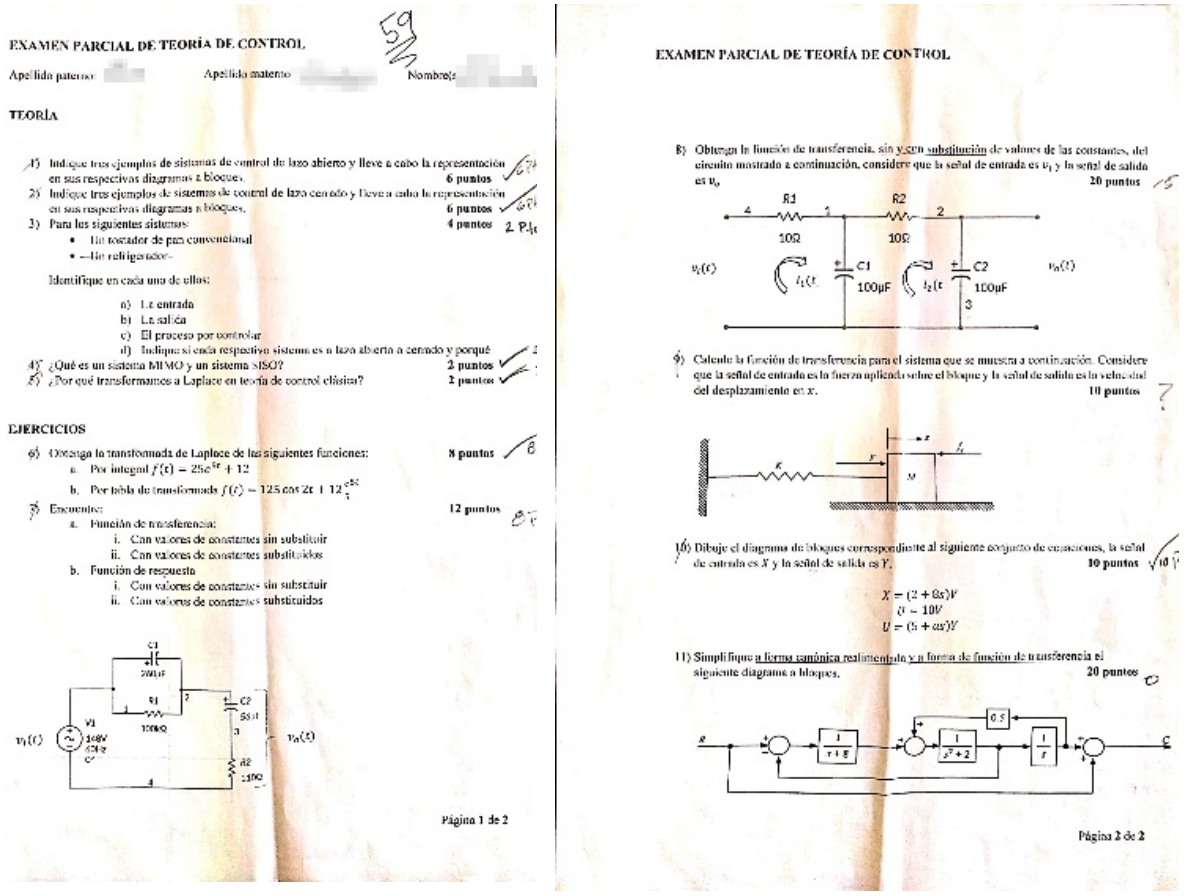

Fig. 2 Muestra de la primera evaluación

La primera evaluación aplicada tiene las siguientes características:

- El examen de evaluación es sobre papel por el método tradicional.

- Consta de dos partes: cinco preguntas teóricas y cinco problemas.

- De los cinco problemas, dos de ellos requieren que el alumno ponga en práctica de manera extensiva las competencias adquiridas. En ese parcial, los otros tres problemas requieren que el alumno haga uso de conocimientos y habilidades a nivel menos complejo.

- El profesor resuelve el examen completo en la siguiente sesión de clase, los entrega y cada alumno y este procede a autocalificarse conforme el profesor va resolviendo el examen.

- El profesor retira los exámenes, y posteriormente revisa que los alumnos se hayan calificado correctamente, hace públicas las notas a través de un aviso en Google Classroom. La Figura 3 muestra el anuncio realizado a través de Google Classroom con la publicación de resultados.

La segunda evaluación consta de una sección desprendible, que contiene una matriz de respuesta como se aprecia en la Figura 4, con la cual el estudiante por instrucciones del profesor ha de registrar sus respuestas en un formulario de Google administrado desde Google Classroom. 


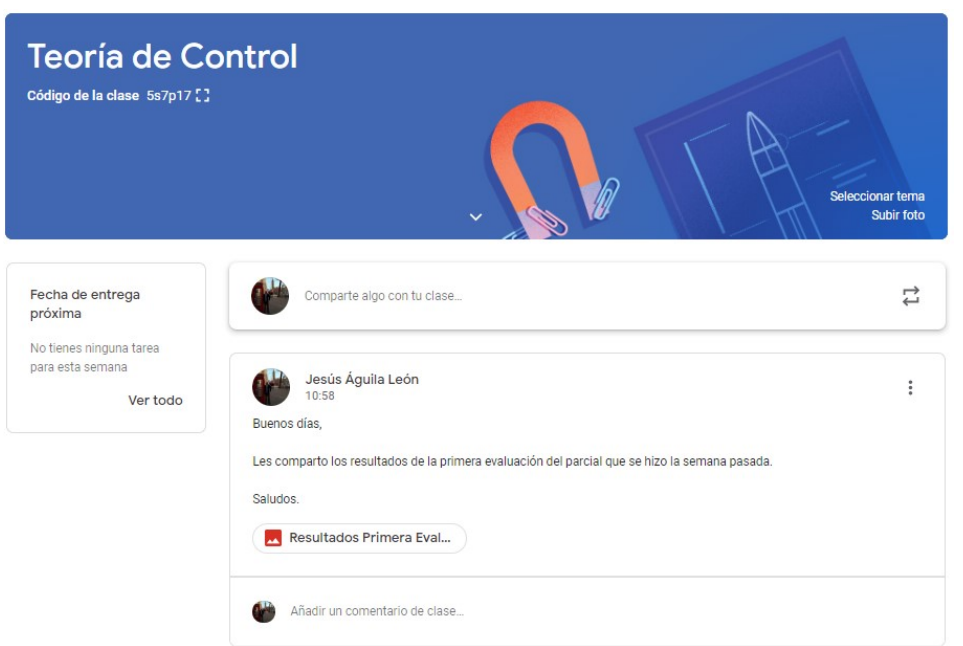

Fig. 3 Captura de pantalla del anuncio con resultados de la primera evaluación.
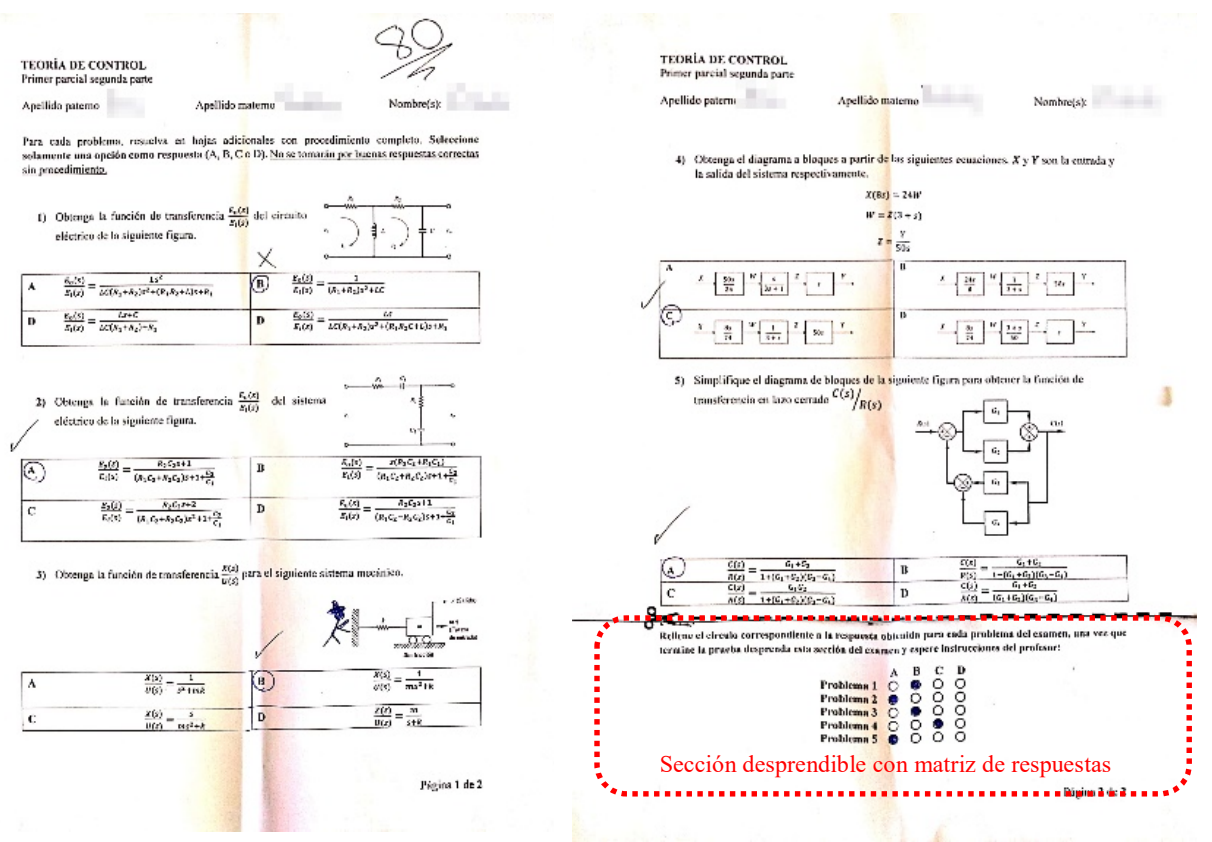

Fig. 4 Plantilla de la segunda evaluación, se aprecia la sección desprendible con la matriz de respuestas

Por su parte, la segunda evaluación tiene las siguientes características principales y se efectúa según se explica a continuación:

- Es de opción múltiple, cinco problemas y cada uno tiene cuatro posibles respuestas, con una posible correcta solución. 
- Dos de los problemas requieren que el alumno haga uso extensivo de las competencias adquiridas, es decir, la aplicación holística de conocimientos, técnicas y habilidades para la resolución de un problema de complejidad moderada.

- El examen es sobre papel, con una matriz para selección de respuestas al final de la prueba.

- El alumno llega a la respuesta por medio de un procedimiento, y debe apuntar la respuesta en la matriz de resultados.

- Una vez ha terminado de contestar, el alumno entregará al profesor la prueba junto con las hojas donde ha llevado a cabo el procedimiento, desprendiendo la sección de la matriz de resultados.

- Los estudiantes tendrán un tiempo límite para registrar sus resultados, registrados en la matriz de respuestas, a través de un Formulario online diseñado en Google Classroom.

- El alumno recibe una nota rápida en base a los resultados registrados en el formulario online.

- El profesor revisa la coherencia entre procedimientos y respuestas anotadas, corrige cualquier discrepancia.

Una vez que el estudiante ha acabado el examen y se ha retirado del aula conserva la sección desprendible del examen con la matriz de resultados, deberá registrar las respuestas en el formulario online indicado por el profesor. La Figura 5(a) muestra el formulario online para registro de datos del estudiante y en la Figura 5(b) se muestra el formulario para completar las respuestas. La autoevaluación pretende que el alumno tenga una actitud proactiva en su proceso de aprendizaje, al reconocer sus propios errores, favoreciendo el subsanamiento de las carencias detectadas, y una mayor participación de los estudiantes en el proceso de evaluación.

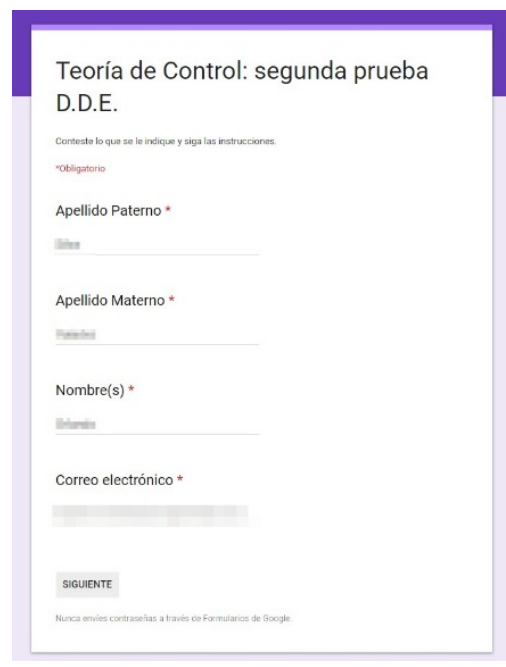

(a)

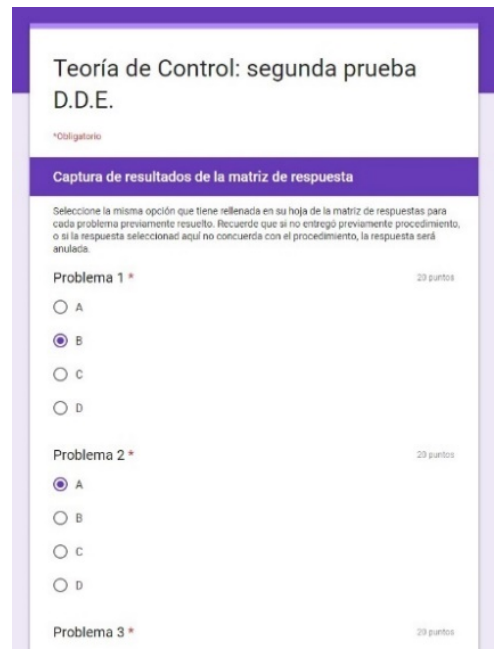

(b)

Fig. 5 Formulario online de Google, (a) datos del alumno y (b) formulario donde se debe introducir la matriz de resultados realizada durante el examen. 


\subsection{Caso de estudio: asignatura de Teoría de Control}

Para evaluar la metodología modificada DDE, esta se aplicó a un grupo de 47 alumnos para la evaluación de su primer parcial del semestre en curso 2019-A, los resultados se compararon contra un grupo de control de 27 alumnos evaluados bajo el esquema tradicional para su primer parcial. La Tabla 1 muestra los contenidos temáticos evaluados durante el primer parcial, para ambos grupos. El grupo de control se evaluó mediante el mismo examen diseñado para la primera evaluación del grupo de DDE modificada.

Tabla 1. Contenidos temáticos evaluados

\begin{tabular}{c}
\hline Teoría de Control: Primer Parcial \\
\hline Introducción a sistemas de control \\
Modelado matemático de sistemas \\
Transformada de Laplace \\
Diagramas de bloque \\
Función de transferencia y función respuesta de sistema
\end{tabular}

Tanto la hoja de preguntas como la hoja de respuestas se diseñaron de tal manera que resultaran intuitivas para el alumno. Como se mencionó anteriormente, al final de la prueba el alumno se queda con la hoja de respuestas y posteriormente se le dio un día como fecha límite para acceder a un formulario creado en la suite de Google Classroom, registrar sus respuestas y obtener así una evaluación rápida de dicha prueba. Una vez que los estudiantes terminan de registrar sus respuestas, el profesor puede acceder a la información estadística y de análisis de los resultados. La Figura 6(a) muestra una captura de pantalla del resumen estadístico que el formulario puede generar y la Figura 6(b) los resultados exportados a una hoja de cálculo. Esta información se analizará en apartado de resultados.

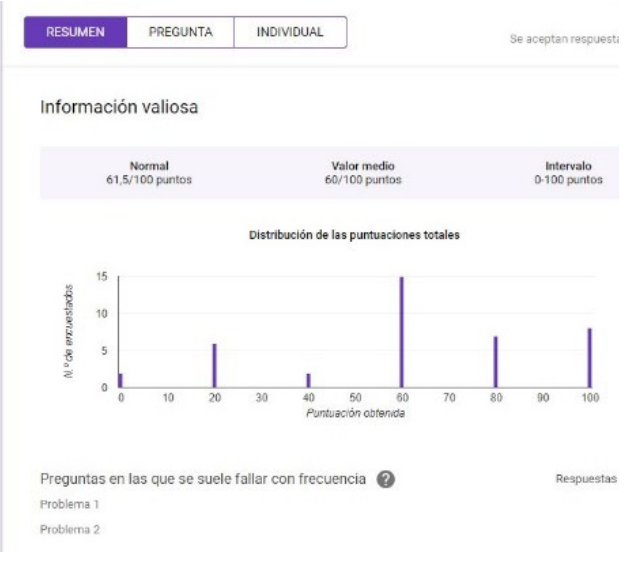

(a)

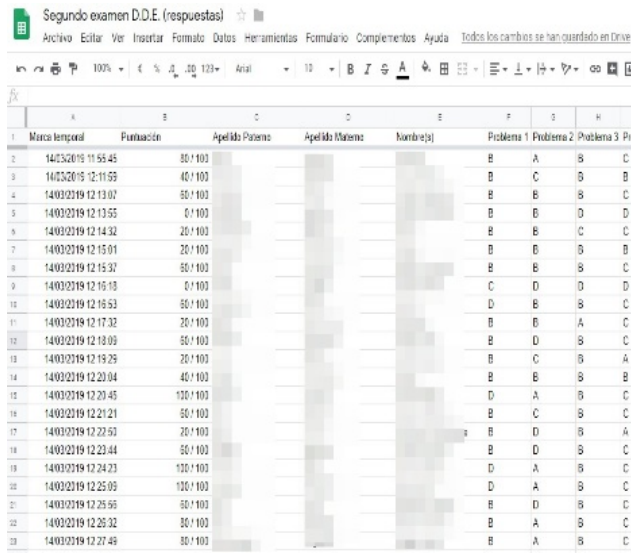

(b)

Fig. 6 (a) Resumen estadístico de respuestas en Google Forms y (b) datos exportados a hoja de cálculo 


\section{Resultados}

Los resultados obtenidos por aplicar la metodología modificada DDE con integración de Google Classroom al grupo de Teoría de Control fueron comparados con los obtenidos por un grupo de control que fue evaluado bajo el esquema tradicional de solo una evaluación por sección del cuatrimestre 2019-A. Para ambos grupos se evaluaron los mismos temas. Adicionalmente se comparan los resultados obtenidos de la metodología modificada DDE contra la metodología DDE presentada por (Boigues Planes et al., 2018).

La Tabla 2 muestra las frecuencias absolutas y relativas de las notas obtenidas por los estudiantes. Es notable como el rango de calificaciones obtenidas entre 60 a 70, 80 a 90 y de 90 a 100 son superiores en un $9 \%, 14 \%$ y 16\%, respectivamente, en el grupo al cual se aplicó metodología DDE modificada en comparación el con el grupo al cual no se le aplicó.

Tabla 2. Notas obtenidas en grupo de control y grupo de metodología DDE con Google Classroom

\begin{tabular}{ccccc}
\hline $\begin{array}{c}\text { Nota obtenida (en } \\
\text { base 100) }\end{array}$ & $\begin{array}{c}\text { Grupo con evaluación tradicional } \\
\text { Frecuencia } \\
\text { absoluta }\end{array}$ & $\begin{array}{c}\text { Frecuencia } \\
\text { relativa }\end{array}$ & $\begin{array}{c}\text { Frupo metodología DDE con Google } \\
\text { Classroom } \\
\text { absoluta }\end{array}$ & $\begin{array}{c}\text { Frecuencia } \\
\text { relativa }\end{array}$ \\
\hline $0-20$ & 0 & $0 \%$ & 1 & $2 \%$ \\
$20-40$ & 1 & $4 \%$ & 10 & $21 \%$ \\
$40-60$ & 11 & $41 \%$ & 5 & $11 \%$ \\
$60-70$ & 5 & $19 \%$ & 13 & $28 \%$ \\
$70-80$ & 7 & $26 \%$ & 0 & 0 \\
$80-90$ & 2 & $7 \%$ & 10 & $21 \%$ \\
$90-100$ & 1 & $4 \%$ & 8 & 17 \\
\hline
\end{tabular}

El grupo DDE se sometió a la metodología descrita en el apartado 3 del presente trabajo, y la calificación final se calculó con la máxima obtenida por el estudiante en cualquiera de las dos pruebas. La Figura 7 muestra la evolución del porcentaje de aprobados y suspendidos en el grupo de metodología DDE modificada. 


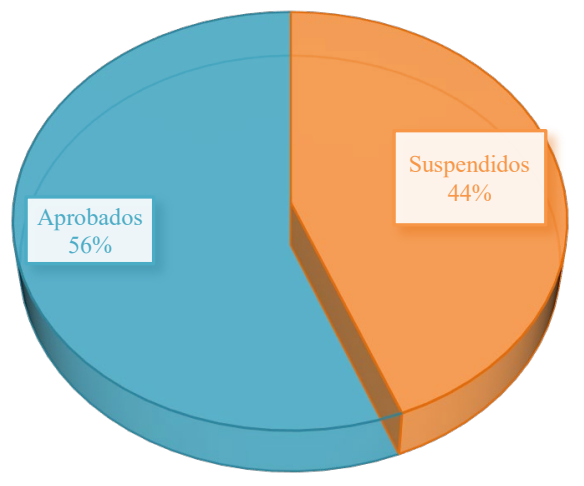

(a) Primera evaluación

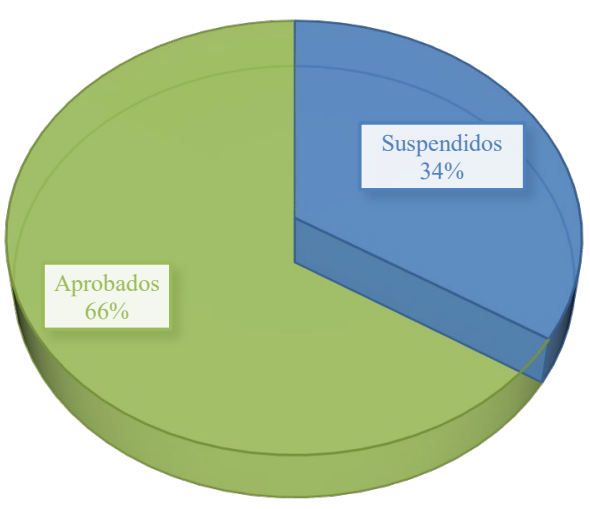

(b) Segunda evaluación

Fig. 7 Evolución del porcentaje de estudiantes suspendidos y aprobados en (a) la primera y (b) segunda evaluación para el grupo con metodología DDE modificada.

La comparativa final, entre el grupo de control, evaluado por el método tradicional, y el grupo evaluado por DDE modificada se muestra en la Figura 8, se aprecia una mejora en el rendimiento de estudiantes, puesto que el porcentaje de alumnos suspendidos es $10 \%$ menor en el grupo con DDE comparado contra el grupo de control, mientras que el porcentaje de aprobados es $10 \%$ superior en la misma comparación.

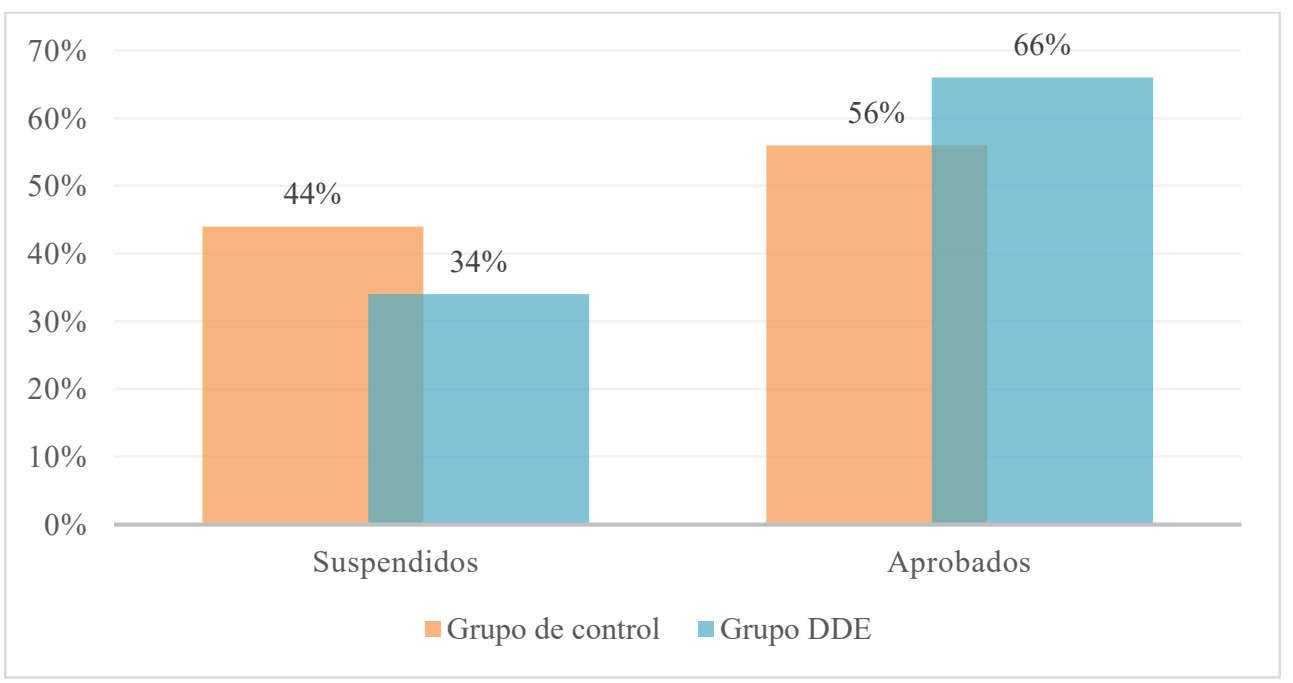

Fig. 8 Comparativa de porcentaje de alumnos suspendidos y aprobados entre el grupo de control evaluado por el método tradicional y el grupo DDE modificada 


\section{Conclusiones}

El presente documento presenta una modificación de la metodología planteada por (Boigues Planes et al., 2018), donde se integra el concepto de autoevaluación del estudiante, tanto en una primera como en una segunda evaluación por medio de un formulario online utilizando la suite de Google Classroom. La metodología aplicada permite el desarrollo de competencias como la comprensión y el pensamiento crítico de los estudiantes aplicando recursos tecnológicos para la docencia como Google Classroom.

Los resultados muestran una mejora en la calificación para el grupo sujeto a la metodología DDE modificada, algo que podría esperarse teniendo en cuenta que es la segunda evaluación de un mismo tema y el estudiante sabe en qué ha fallado antes, sin embargo, para evitar este tipo de situación la metodología DDE modificada plantea que la segunda evaluación del proceso sea diferente, pasando de un examen de problemas abiertos a un examen de opciones cerradas, donde el estudiante se encuentra con problemas con retos mayores y por tanto más difíciles que los de la primera evaluación.

Las mejores notas fueron obtenidas para el caso del grupo de DDE modificada, donde el porcentaje de aprobados fue de $66 \%$ comparado contra el 56\% del grupo evaluado de forma tradicional. Para el grupo que se aplicó la metodología DDE el aumento de porcentaje de alumnos aprobados fue de $66 \%$, pasando de $19 \%$ a $64 \%$ entre la primera y la segunda evaluación respectivamente. Por su parte, las peores calificaciones se obtuvieron en la primera evaluación de control para el grupo DDE, donde el $81 \%$ de los alumnos suspendió el examen.

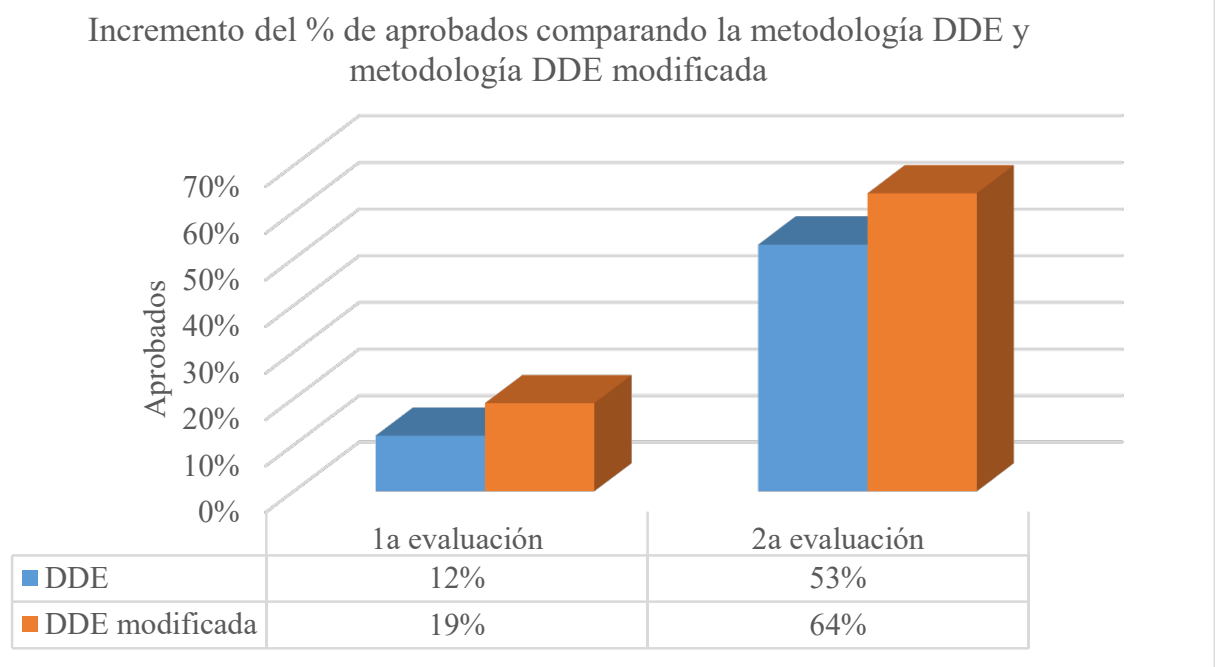

Fig. 9 Comparativa entre el incremento de estudiantes aprobados durante la primera y la segunda evaluación de los resultados de (Boigues Planes et al., 2018) para la metodología DDE y la metodología DDE modificada del presente trabajo.

La comparativa entre la evolución de porcentaje de alumnos aprobados empleando la metodología DDE propuesta por (Boigues Planes et al., 2018) y la metodología modificada DDE propuesta en este trabajo muestra que el hecho de que involucrar al estudiante en el 
proceso de evaluación mediante la auto calificación favorece la autocrítica, ayudando a que el estudiante se motive para la obtención de una mejor nota en la segunda prueba. Puesto que el porcentaje de mejora de calificación mostrado con la metodología modificada fue superior en un $8 \%$ contra la metodología DDE según puede observarse en la Figura 9.

Se deduce que la metodología modificada DDE tiene efectos positivos sobre el estudiante, pues al integrar mecanismos de autoevaluación y autocrítica se ayuda a reforzar el conocimiento adquirido y la experiencia después de haber realizado una primera evaluación, en comparación con los resultados de la metodología DDE presentados por (Boigues Planes et al., 2018) donde los alumnos no se autocalifican ni utilizan herramientas tecnológicas. Mediante la integración de Google Classroom realizada en el presente trabajo se obtiene un mayor involucramiento del alumno y una mejora de los resultados al aplicar una metodología DDE gracias a la auto evaluación, auto crítica y competencia consigo mismo.

\section{Agradecimientos}

Este trabajo ha sido respaldado en parte por la administración pública de Valencia bajo la beca ACIF/2018/106.

\section{Referencias}

ABID AZHAR, K e IQBAL, N. (2018). “Effectiveness of Google Classroom: Teachers' Perceptions " en Prizren Social Science Journal, vol. 2, issue 2, p. 52-56.

$<$ https://doi.org/10.1985/2> [Consulta : 06 de Marzo de 2019]

AL-MAROOF, R. y AL-EMRAN, M. (2018), “ Students acceptance of google classroom: An exploratory study using PLS-SEM approach " en International Journal of Emerging Technologies in Learning, vol. 3, issue 16, p. 112-123.

$<$ https://doi.org/10.3991/ijet.v13i06.8275> [Consulta : 06 de Marzo de 2019]

ANG, K. (2001), "Teaching mathematical modelling in Singapore schools" en The Mathematics Educator, vol. 6, issue 1, p. 63-75.

$<$ https://repository.nie.edu.sg/handle/10497/49> [Consulta : 06 de Marzo de 2019]

BOIGES, F., ESTRUCH, V., VIDAL, A. y PASTOR, J. (2018), “ La doble evaluación: un medio para aprender de los errores y para mejorar el rendimiento en matemáticas ” en IN-RED2018, p. 449-460.

$<$ https://doi.org/10.4995/inred2018.2018.8569> [Consulta : 06 de Marzo de 2019]

BORROMEO, R. (2018). " Key Competencies for Teaching Mathematical Modeling Research in Teacher Education on Mathematical" en Ferri, R. B. Learning How to Teach Mathematical Modeling in School and Teacher Education. Kassel, Germany : Institute of Mathematics, University of Kassel.

FABREGAT, J., PELAYO, I. (2016), “ Plan inicial, progresos explícitos y evaluación de una trama de feedforwarding sobre campus virtual en una asignatura virtual " en IN-RED2016, p. 1056-1068.

GARGALlO, B., GARCÍA, E., MORERA, I. y BENAVENT, A. (2015), “ Métodos innovadores y enfoques de aprendizaje en estudiantes universitarios" en IN-RED2015, p. 47-54. 
Metodología de doble evaluación modificada e integración de entornos virtuales para el proceso de enseñanza y aprendizaje

GREEFRATH, G., VORHÖLTER, K. (2018). "Teaching and Learning Mathematical Modelling : Aproaches and Developments from German Speaking Countires", Hamburg, Germany : Faculty of Education, University of Hamburg.

< https://doi.org/10.1007/978-3-319-45004-9_1 > [Consulta: 06 de Marzo de 2019]

HEGGART, K. R. y YOO, J. (2018). “ Getting the most from google classroom: A pedagogical framework for tertiary educators" en Australian Journal of Teacher Education, vol. 43, issue 3, p. 140153.

< https://doi.org/10.14221/ajte.2018v43n3.9> [Consulta: 06 de Marzo de 2019]

KAISSER, G., BLUM, W., BORROMEO, R., (2011). "Trends in Teaching and Learning of Mathematical Modelling", Springer Netherlands.

$<$ https://www.ellibs.com/sv/book/9789400709102/trends-in-teaching-and-learning-of-mathematicalmodelling $>$ [Consulta: 06 de Marzo de 2019]

KENG, C. (2005). "Mathematical Modelling in Singapore Schools : a Framework", in Mathematical Modelling From Theory to Practice, Series on Mathematics Education, World Scientific.

MAAß, K. (2006), "What are modelling competencies?" en ZDM - International Journal on Mathematics Education, vol. 38, issue 2, p. 113-142.

$<$ https://doi.org/10.1007/BF02655885 > [Consulta: 06 de Marzo de 2019]

RYLANDS, L. J. (2009), “ Performance of students with weak mathematics in first-year mathematics and science" en International Journal of Mathematical Education in Science and Technology, vol. 40, issue 6, p. 741-753.

< https://doi.org/10.1080/00207390902914130 > [Consulta: 06 de Marzo de 2019]

UNIVERSITAT POLITÈCNICA DE VALÈNCIA. PoliformaT: Formación Online Centro de Formación Permanente. < https://formaciononline.blogs.upv.es/poliformat-2/poliformat/> [Consulta: 06 de Marzo de 2019]

YAVICH, R., STARICHENKO, B. (2017), " Design of Education Methods in a Virtual Environnent" en Journal of Education and Training Studies, vol. 5, issue 9, p. 176-186.

$<$ https://doi.org/10.11114/jets.v5i9.2613 > [Consulta: 06 de Marzo de 2019]

ZAWOJEWSKI, J. (2007), “ Modeling Student's Mathematical Modeling Competencies " en International Perspectives on the Theaching and Learning of Mathematical Modelling, p. 237-243.

$<$ https://doi.org/10.1007/978-1-4419-0561-1 > [Consulta: 06 de Marzo de 2019]

(c)) BY-NC-ND 2019, Universitat Politècnica de València 